\title{
Participation transnationale et citoyenneté : les immigrés dans l'Union européenne
}

\section{Riva Kastoryano}

\section{(2) OpenEdition}

1 Journals

\section{Édition électronique}

URL : http://journals.openedition.org/conflits/2121

DOI : 10.4000/conflits.2121

ISSN : $1777-5345$

Éditeur :

CCLS - Centre d'études sur les conflits lilberté et sécurité, L'Harmattan

\section{Édition imprimée}

Date de publication : 15 janvier 1997

ISSN : 1157-996X

Référence électronique

Riva Kastoryano, «Participation transnationale et citoyenneté : les immigrés dans l'Union européenne », Cultures \& Conflits [En ligne], 28 | hiver 1997, mis en ligne le 21 mars 2007, consulté le 30 mars 2021. URL : http://journals.openedition.org/conflits/2121; DOl : https://doi.org/10.4000/ conflits. 2121

Ce document a été généré automatiquement le 30 mars 2021.

Creative Commons License 


\title{
Participation transnationale et citoyenneté : les immigrés dans l'Union européenne
}

\author{
Riva Kastoryano
}

1 Définir l'Union européenne comme une " communauté de destin " ou l'expression d'une " volonté de vivre ensemble "1, renvoie aux idées qui étaient à l'origine de la formation des Etats-Nations européens au XIXe siècle. Peut-on imaginer l'Union européenne dans les mêmes termes que l'Etat-Nation? Les efforts de la part des Etats pour mettre en place un " agenda politique " 2 ou, le souci des institutions européennes à harmoniser les différences culturelles et juridiques, suffisent-ils à concevoir l'Union comme une communauté politique?3

2 Cela pose directement la question des modes de participation politique dans l'Europe unie et celle de la citoyenneté. D'après le traité de Maastricht est " citoyen de l'Union " toute personne qui a la nationalité d'un des Etats membres (art.8). Une telle définition, qui n'est que la projection de la citoyenneté liée à la nationalité et élaborée dans le cadre des Etats-Nations, se trouve en contradiction avec sa pratique, dans la mesure où, toujours d'après le traité de Maastricht, le droit de vote local est accordé aux " citoyens de l'Union " en fonction de leur résidence. Cela introduit d'emblée une séparation entre la citoyenneté (l'exercice de droit) et la nationalité (l'appartenance). Dans cette perspective, la pratique de la citoyenneté fait apparaître une multiplicité d'appartenances et d'allégeances pour le citoyen de l'Union et constitue de fait un défi à l'Etat-Nation.

Quant aux populations issues de l'immigration des années 1960, dont certains membres d'origine non-européenne sont parfois citoyens de leur pays de résidence, d'autres fois juridiquement étrangers, une participation politique de nature transnationale se heurte de la même façon aux limites territoriales de l'action politique et de la citoyenneté. En effet, même si les politiques d'immigration et d'intégration relèvent de la compétence des Etats, les populations issues de l'immigration développent des réseaux de solidarités " primordiales " qui traversent les frontières en fonction d'une 
ou des identités dites " d'origine ". Ces réseaux, tout comme ceux des groupes professionnels, forment les fils d'une toile d'araignée couvrant l'espace européen, cet " espace sans frontières intérieures dans lequel - selon l'Acte Unique Européen de 1986 la libre circulation des marchandises, des biens et des capitaux est assurée ".

Une organisation transnationale pourrait permettre aux populations issues de l'immigration de contourner les politiques nationales, et d'aboutir à une reconnaissance au-delà des limites établies par les modèles nationaux. Elle traduit ainsi la recherche d'une représentativité au niveau européen. Cette évolution met surtout en évidence les multiples interactions entre les sociétés nationales et l'espace européen comme espaces de participation politique, ainsi qu'entre Etats-Nations et institutions européennes supranationales. La complexité des situations conduit à une nouvelle conception de la citoyenneté partagée entre le pays d'origine, le pays de résidence et l'Europe. Ce qui conduirait à redéfinir le lien entre la citoyenneté, la nationalité et l'identité à la fois dans le cadre de l'Etat-Nation et de l'Europe unie.

5 Ainsi, la recherche d'un nouveau modèle politique pour l'Europe unie s'éloigne du modèle de la formation de l'Etat-Nation et lui pose au contraire un défi. S'interroger sur les formes d'organisation des populations immigrées et sur l'expression de leurs identités collectives, c'est aussi s'interroger sur les traditions politiques, sur les " modèles " nationaux redéfinis en réaction à la fois à l'immigration et à la construction européenne. Dans quelle mesure, une action politique au niveau européen constitue-telle, pour chacun des Etats-Nations, une pression poussant à redéfinir leurs relations avec les populations " immigrées "?

Solidarités et identités transnationales

6 Tous les pays européens sont devenus des pays d'immigration de fait. Certains depuis longtemps comme la France, d'autres depuis les années 1960, comme l'Allemagne, la Grande-Bretagne, et d'autres encore depuis une période récente comme l'Espagne ou l'Italie. Ce sont les relations, a priori, historiques et politiques entre les pays de départ et les pays d'arrivée qui ont décidé des destinations et de l'installation des différentes populations du Sud ou de l'Est de la Méditerranée, d'Afrique ou de la péninsule indienne dans différents pays membres de l'Union4.

7 Chacun des pays cherche à mettre en évidence ses spécificités puisées dans les principes fondateurs des Etats-Nations, et souligner ainsi ses divergences quant à ses relations avec l'immigration et avec la présence des populations immigrées5. Dans cette perspective, le modèle français est défini comme l'exemple même d'Etat-Nation fondé sur l'individualisme républicain ayant comme " doctrine " l'assimilation des individus devenus citoyens par choix politique. Ce modèle s'oppose à l'Allemagne par sa conception élective et politique de la nation contre une conception culturelle et ethnique de celle-ci qui privilégie les ancêtres communs. Quant au modèle dit anglosaxon, il se caractérise, au regard du modèle français, par la reconnaissance des communautés culturelles, nationales, religieuses dans la vie publique.

8 Ces différences dans les traditions politiques de chacune des nations se répercutent dans l'expression des identités collectives et des revendications des populations issues de l'immigration. En effet, leurs demandes sont, dans la plupart des cas, définies en réaction à l'identité nationale et permettent de cristalliser les éléments autour desquels pourrait se structurer une communauté. En France, par exemple, les associations dites d'immigrés rejettent l'idée de toute intervention dans le domaine " ethnique ", terme utilisé dans le cadre britannique. Les discours des militants renvoient aux 
préoccupations de l'Etat qui cherche à mettre en avant l'exclusion sociale. En GrandeBretagne, les communautés ethniques se reconnaissent dans le développement de l'identité " Black " résultant de la lutte contre le racisme. En Allemagne, l'" ethnicité " devient synonyme de nationalité en référence à la revendication de la double nationalité, source d'une action collective.

Mais, en même temps, se dessine une convergence entre différents pays européens quant aux stratégies et aux modes de participation politique des acteurs politiques issus de l'immigration. Que les Etats se définissent comme républicains et/ou qu'ils favorisent la constitution de communautés ethniques, les stratégies d'intégration deviennent de plus en plus collectives, privilégiant une appartenance culturelle. Cela n'empêche pas cependant que ces " communautés " entretiennent des relations avec les institutions nationales et/ou locales dans chacun des pays.

Cela est en grande partie le résultat d'une convergence qui se situe au niveau des politiques dites d'intégration et de la gestion des identités collectives qui émergent dans l'espace public. S'inspirant d'un certain libéralisme, les Etats européens s'appuient sur la démocratie comme méthode, afin de définir des mesures politiques spécifiques pour intégrer les " différences " dans la communauté nationale.

11 Ces convergences parallèles se rejoignent dans l'espace européen pris comme un nouvel espace politique. En effet, l'espace de l'Union réunit les actions à la fois des Etats-Nations et celles des acteurs politiques de l'immigration qui cherchent à marquer leur indépendance par rapport aux " modèles " nationaux. Des populations " immigrées/issues de l'immigration " ou " étrangères " ou encore des " communautés ethniques " - selon la terminologie de chacun de ces pays - se réfugient dans des réseaux de solidarités transnationales fondées sur la nationalité ou la religion ou les deux. Même si les politiques d'immigration et d'intégration relèvent toujours des compétences nationales, elles cherchent un point d'appui dans ce nouvel espace politique à l'identité incertaine qu'est l'Europe. C'est ce qui est apparu spécialement au moment de la signature du traité de Maastricht, quand, en mettant en évidence une volonté de dépassement des cadres nationaux européens, certains parlaient de "13ème population " ou de " 13ème Etat ", ou encore de " 13ème nation " pour souligner une forme d'organisation " communautaire transnationale ".

Certains des réseaux qui alimentent cette vision transfrontière émanent d'initiatives locales, tandis que d'autres sont mis en place par des institutions de l'Union européenne, notamment par le Parlement européen, et d'autres encore sont encouragés par les pays d'origine. Mais tous aident les militants à élaborer des stratégies qui dépassent les systèmes étatiques. " Il faut prendre des habitudes, il faut s'adresser à des organisations supranationales, arriver à un organisme qui sera à Strasbourg, à Bruxelles, qui sera européen, et qui devra trancher " dit la responsable d'une association à Marseille.

13 Les institutions européennes interviennent pour définir un dénominateur commun qui tiendrait compte des revendications au-delà des relations avec les Etats-Nations. C'est ainsi que, depuis 1986, le Parlement européen met à la disposition des associations dites d'immigrés des fonds pour qu'elles puissent coordonner leurs activités. C'est de cette initiative qu'est née une nouvelle structure transnationale appelée " Forum des migrants ". Bien que le Forum doive sa création à la politique budgétaire de l'Union, il vise néanmoins à devenir " un lieu d'expression pour les populations non communautaires établies en Europe, à travers lequel elles puissent faire part de leurs 
revendications, mais aussi diffuser l'information émanant des instances européennes "6. D'après le responsable chargé du suivi du Forum des migrants à la Commission des Communautés Européennes, le but serait d'obtenir pour les ressortissants des pays tiers " les mêmes opportunités et les mêmes droits que les autochtones, et de compenser ainsi le manque démocratique ". Explicitement, l'objectif est donc de s'opposer de façon légale à la montée du racisme dans les différents pays européens. Par conséquent, ce sont surtout des associations dont les activités sont les plus soutenues par les Etats des pays membres, et dont les dirigeants élaborent un discours sur l'égalité des droits, sur les Droits de l'Homme et leur universalité qui voient dans la mobilisation transnationale une façon efficace de lutter contre le racisme et la xénophobie7.

Le Forum des migrants rassemble en principe, les associations d'immigrés formées avant tout en vertu du critère de la nationalité. Mais, en même temps, la capacité d'organisation mesurée en termes de multiplicité des nationalités représentées, de nombre de filiales, d'étendue des réseaux, de pluralité des secteurs qu'elles recouvrent (économique, social, culturel), et, bien entendu, de leur représentativité dans le pays où elles se situent, constituent des critères de choix d'associations membres du Forum.

En réalité, le critère principal de structuration de réseau se résume à l'identité. Des identités présumées d'origine, ou des identités de " circonstance ", pour reprendre le terme de Jean Leca, construites en relation avec les Etats d'accueil, institutionnalisées par les associations forment en fait les maillons de la chaîne transfrontalière. Cela est confirmé par les actions locales de certaines associations, puisqu'elles comprennent désormais des programmes d'échanges culturels, de concerts, de débats à travers l'Europe pour définir leur position sociale et légale dans l'Union. L'objectif déclaré des instances européennes les obligent dans les faits, à dissimuler leurs préoccupations identitaires dans leurs stratégies de reconnaissance en tant qu'" Européens noneuropéens".

16 Ainsi apparaît le paradoxe de la supranationalité8. En effet, les institutions européennes constituent par essence un défi aux Etats-Nations. Avec la création du Forum des migrants, le Parlement européen marque son autonomie par rapport aux institutions nationales, et incite les acteurs issus de l'immigration à se situer au-delà des Etats-Nations grâce à une action transnationale. Mais en même temps, en définissant les critères d'adhésion au Forum des migrants dans les mêmes termes que ceux des Etats Providence des pays membres, le Parlement européen projette les représentations des identités collectives au niveau européen. Derrière le critère de nationalité comme élément identitaire qui se veut a priori objectif, puisque défini juridiquement, font partie du Forum des migrants des associations qui sont fonction de la " nationalité d'origine " (tel est le cas des associations de jeunes issues de l'immigration maghrébine en France, ou de celles des Blacks en Grande-Bretagne; les premières représentant des jeunes de nationalité française pour la plupart, et les secondes de nationalité britannique), selon la religion, selon la " couleur " de la peau. Il s'agit donc non de nationalité mais d'ethnicité.

17 Dans cette perspective, l'espace européen apparaît comme la projection de l'espace national à l'échelle transnationale. Déjà au niveau national, la lutte contre le racisme est de plus en plus liée à l'affirmation d'une identité collective face aux problèmes de société, perçus et vécus similairement, en raison d'une communauté d'origine et du développement d'une " ethnicité " fondée sur des bases identiques. 

deuxième étape de socialisation politique, la première étant celle opérée dans le cadre des sociétés nationales. En effet, depuis les années 1980, grâce à la prolifération d'associations dites d'immigrés créées et soutenues par les pouvoirs publics, se sont exprimées des identités religieuses, nationales, ethniques pour se positionner face à l'Etat en vue d'une reconnaissance.

19 Une participation accrue à la fois aux associations et aux institutions globales où s'acquiert la " vertu civique " inaugure l'exercice même de la citoyenneté, et plus encore la formation d'une " identité de citoyen ". Elle s'exprime à la fois dans les institutions dites communautaires et nationales, et avant tout, met en évidence l'engagement de l'individu9. Cela n'est en somme que le résultat d'un processus d'" acculturation politique ", c'est à dire de l'intériorisation des valeurs nationales du pays d'accueil et de ses règles du jeu politique.

Les concepts mêmes de citoyenneté et de nationalité se définissent avant tout par l'appartenance à une communauté politique. Une " identité de citoyen " dérive surtout de la participation, qu'elle soit directe ou indirecte, à la vie publique des immigrés comme du gros de la population. Bien entendu les lois sur la nationalité affectent les modes de participation et les stratégies des acteurs. Par exemple, la citoyenneté définie en termes politiques, et donc juridiques, des jeunes issus de l'immigration en France les conduit à participer directement dans la communauté nationale par le vote. Alors qu'en Allemagne, où les lois sur la nationalité privilégient les relations de sang et non la naissance sur le sol national comme en France, les " étrangers " élaborent des stratégies de compensation par des voies indirectes de participation, poussant les " étrangers " à trouver des moyens d'influencer l'opinion publique. A travers les associations, ils s'intègrent avant tout dans le système corporatiste et, par conséquent, dans la société civile. Apparaît alors une citoyenneté qui pourrait être qualifiée de civile, par opposition à une citoyenneté civique. Ainsi, les immigrés, juridiquement citoyens ou étrangers, sont conduits à agir dans l'espace public, cet espace commun de socialisation et d'exercice du pouvoir, et manifester ainsi leur engagement et leur appartenance au moins de fait à la communauté nationale.

21 Une telle définition de la citoyenneté va à contre-courant des analyses classiques de la citoyenneté systématiquement rattachée au cadre de l'Etat-Nation, où se confondent ses aspects identitaires et politiques. Les registres d'appartenance et d'engagement politique montrent que la pratique de la citoyenneté se détache d'une conception exclusivement liée à l'identité nationale. Que la citoyenneté soit politique, juridique, sociale, économique, et son contenu identitaire, culturel ou juridique, cette combinaison se résume en un sentiment de loyauté dirigé à la fois vers le groupe, la communauté, la société civile et l'Etat.

Ainsi, la réalité sociale tend à bouleverser l'imbrication de la communauté nationale et de la communauté politique, le lien entre l'identité et le droit, ainsi que celui entre la culture et la politique. Par conséquent, la citoyenneté se limite de plus en plus à un droit de participation civique, et n'exclut plus, toujours en théorie, l'expression des identités collectives, même si cela remet en cause l'idéologie républicaine. Cependant, malgré les différences de traditions politiques qui privilégient en France la participation à une communauté politique dite républicaine, alors que celle-ci s'oriente vers la société civile en Allemagne, il s'agit dans les deux cas du dépassement d'un concept de citoyenneté strictement limité dans le cadre de l'Etat-Nation, par 
conséquent d'une dissociation de la citoyenneté de la nationalité, qui réduit la première aux droits politiques et donne à la seconde sa dimension purement identitaire. La multiplicité des identifications et des allégeances culturelles, ethniques ou religieuses qui en découle conduit à de nouvelles définitions de l'appartenance qu'on qualifie maintenant de postnationale et qui trouve surtout une signification dans les projets de construction d'une Europe politique.

Une identité de citoyen postnational

Avec les réseaux de solidarité transnationaux, une nouvelle pratique de participation politique s'introduit dans l'Europe unie comme dans les Etats-Nations. Les acteurs non nationaux, résidents européens, affirment à travers elle, leur autonomie par rapport aux systèmes étatiques territorialement définis. En revendiquant l'égalité des droits et de traitement, ils s'efforcent de promouvoir leur statut de " citoyen européen " dans le nouvel espace politique en formation.

De nombreux débats sur la citoyenneté, la nationalité et l'identité européenne ont accompagné la transformation progressive d'un grand marché unique en un espace politique. Jean-Marc Ferry propose un modèle " postnational " pour souligner le dépassement $d u$ " principe nationaliste " entraîné par la construction d'une Europe politique10. Habermas, de son côté, développe le concept de " patriotisme constitutionnel " pour souligner la séparation entre le sentiment d'appartenance qu'implique la citoyenneté nationale et sa pratique juridique au-delà des cadres de l'Etat-Nation11. De même, considérant les populations non-européennes issues de l'immigration des années 1960, Yasemin Soysal définit comme " postnationale " l'adoption de normes internationales se référant à la personne ou à la résidence et non à la citoyenneté juridique 12 .

Cette conception postnationale de l'appartenance alimente surtout les discours normatifs touchant à la définition nécessaire d'un nouveau modèle de citoyenneté. Mais, les projets européens ne vont pas toujours dans le sens de ces discours. Du point de vue juridique, le traité de Maastricht maintient le lien entre la citoyenneté nationale et la " citoyenneté de l'Union ". Le citoyen de l'Union a le droit de circuler et de séjourner librement sur le territoire des Etats membres, et même le droit de voter aux élections municipales et européennes dans un Etat membre dont il n'est pas le ressortissant, mais simplement un résident. Cette pratique introduit de fait, une notion d'extraterritorialité de la citoyenneté, et remet en cause l'adaptabilité des citoyennetés nationales au sein des cadres juridiques européens.

Quant à l'engagement politique des militants de l'immigration pour une égalité des droits au niveau national et européen, il s'accompagne chez eux d'un langage nouveau en ce qui concerne la citoyenneté. " Nous sommes des citoyens européens ; nous faisons partie du paysage européen ", dit un responsable d'association. Dans ce domaine, le paysage européen n'est rien d'autre pour eux que cette toile d'araignée, formée de réseaux de solidarité et d'intérêts, étalée sur un territoire qui recouvre maintenant 15 pays. Leur présence dans cette toile leur confère, à leurs yeux, un " droit " de participer à la formation de l'Europe, justifié par leur engagement en faveur d'un nouvel espace politique qui offrirait à tous un statut juridique de citoyen de l'Union ou, au moins, de résident européen installé dans un des pays membres depuis souvent près de trente ans.

La structuration des réseaux transnationaux de la part des " résidents européens " traduit une identification avec la société européenne. La citoyenneté implique alors, de 
la part des " non nationaux " d'un des pays membres, une responsabilité dans la formation de la nouvelle " communauté de destin ". Les militants associatifs qui se posent comme résidents européens ont cependant du mal à définir le statut qui leur convient. " Nous sommes quelque part européens, citoyens de l'Europe. Si nous ne sommes pas des citoyens européens, nous nous considérons en tout cas comme des citoyens d'Europe ", dit un responsable d'association. " Autant pour l'acquisition de la nationalité, nous, nous aurions préféré qu'il y ait une nationalité européenne en ce qui concerne la libre circulation ", ajoute-t-il. Alors que la nationalité dans le cadre d'un Etat-Nation est porteuse d'identité et d'une citoyenneté de droit, dans le discours de ce militant, cette dernière revêt une portée juridique en ce sens que l'accès à la citoyenneté de l'Union devrait entraîner le droit de libre circulation qui lui est rattaché.

Cette confusion est significative ; imprégnée par une tradition républicaine, elle se réfère à la citoyenneté sur une base culturelle et à la nationalité comme source de droit. Telle est aussi la conception d'Alain Touraine quand il écrit, " je souhaite moi-même acquérir une nationalité européenne et conserver une citoyenneté française ", en émettant aussi l'hypothèse d'un Etat européen. En fondant son raisonnement sur cette séparation entre Etat et société, Touraine lie la nationalité à l'Etat et la citoyenneté à la société13. Mais en détachant la nationalité de la citoyenneté, indissociables dans la logique républicaine, il côtoie une tradition libérale d'après laquelle, selon Habermas, la citoyenneté " est conçue sur le modèle d'affiliation à une organisation qui assure une position juridique et situe l'individu en dehors de l'Etat "14.

Ainsi, les discours sur l'Europe politique s'accumulent, se ressemblent ou se contredisent. Leur polysémie met en évidence le bouleversement du " modèle " de l'Etat-Nation et la difficulté de s'en détacher, tout en soulignant son absence de compatibilité avec la réalité européenne, où une citoyenneté " postnationale " est loin de se dessiner. L'Europe se bâtit plutôt sur les institutions supranationales dont la conception et le fonctionnement s'opposent au concept de postnational. Alors que ce dernier amènerait à reconnaître la diversité culturelle et à accepter le pluralisme comme le fondement de l'appartenance européenne, le supranational apparaît comme une projection de l'Etat-Nation et s'impose aux Etats. Plus encore, tout en remettant en cause l'Etat-Nation, il renforce le rôle de l'Etat dans la construction d'une Europe politique.

" Bringing the State Back in "

31 La consolidation des solidarités transnationales vise en somme à influencer les Etats par l'extérieur. Même si les réseaux transnationaux contribuent, à certains égards, à la formation de " communautés à part ", ces dernières apparaissent désormais comme des structures indispensables pour négocier avec les pouvoirs publics la reconnaissance d'identités collectives construites dans des cadres qui restent nationaux. En effet, la structuration transfrontalière des réseaux associatifs a pour objectif de renforcer leur représentativité au niveau européen, mais son but pratique est d'aboutir à une " reconnaissance " au niveau national. Faut-il préciser que les militants, même les plus actifs au niveau européen, se représentent les Etats comme les seuls " adversaires " avec lesquels ils doivent compter en dernière instance? Cette prédominance des Etats se fait d'ailleurs sentir dans la difficulté qu'ont les associations à coordonner leurs actions et leurs revendications, lorsque cela émane de leur propre initiative. 
32 Autrement dit, il s'agit d'aboutir à une représentativité politique qui ne peut se définir que nationalement pour défendre des droits au séjour, au logement et à l'emploi, liés à la prise en compte de cette identité. Ainsi, toute revendication à un niveau national sous-entend désormais une pression parallèle au niveau européen. Mais inversement, toute revendication au niveau européen vise à avoir des effets sur les décisions nationales de chacun des Etats membres : " pour nous, immigrés des pays tiers, il faut faire en sorte qu'effectivement nous puissions nous organiser et nous défendre, porter haut nos revendications ; parce que la plupart de nos recommandations qui sont reprises par la CEE, et qui nous sont souvent favorables ne sont pas toujours bien vues par les pays membres... Faisons en sorte que ce qui est positif à l'échelle européenne puisse se répercuter dans le pays " (citation des propos d'un responsable de l'Union des travailleurs africains en France).

33 Ainsi, l'Europe unie introduit un " supranationalisme normatif "16 qui dépasse le cadre des Etats-Nations, voire s'impose aux Etats. En cas d'expulsion, par exemple, l'étranger peut s'opposer aux décisions nationales au nom du droit au respect à la vie familiale (art.8 §1), après avoir épuisé les voies de recours internes. Même si les Droits de l'Homme demeurent de la compétence exclusive des Etats, ces derniers se trouvent contraints d'accepter les nouvelles normes juridiques produites par les institutions européennes, dans la mesure où la Convention Européenne des Droits de l'Homme autorise le citoyen européen (dans ce cas celui qui a la nationalité d'un des Etats qui a accepté le recours individuel) à saisir directement le Conseil de l'Europe, et un étranger (qui n'a pas la nationalité d'un des pays de l'Union) à avoir recours à la Cour Européenne des Droits de l'Homme.

Par ailleurs, le droit des " droits de solidarité "17, se référant à la liberté de l'action collective dans un cadre communautaire et affirmant que " c'est seulement dans la communauté que le plein développement de la personnalité de l'individu est possible "18, pourrait renvoyer, dans le cas des populations issues de l'immigration, au droit de " minorité ". D'après la Convention des Droits de l'Homme, " le terme minorité désigne un groupe numériquement inférieur au reste de la population et dont les membres sont animés de la volonté de préserver leur culture, leurs traditions, leur religion ou leur langue "19.

35 Ce concept idéologiquement chargé suscite des réactions qui varient d'un pays à l'autre. En France, qu'il s'agisse des identités régionales ou religieuses, ou encore des identités collectives exprimées par les populations issues de l'immigration, le terme est rejeté. En Allemagne, il renvoie seulement aux minorités allemandes installées hors du territoire allemand. Dans tous les cas, l'usage officiel du terme inspire notamment les ressortissants turcs qui expriment la volonté de structurer une communauté nationale turque ou kurde en Allemagne. La réorganisation de leurs associations dans ce sens conduit la République fédérale à réagir dans les mêmes termes.

36 Un espace supranational qui accroît les interactions entre les pays donne lieu à des résistances auxquelles se heurte l'unification européenne et qui, en réalité, soulignent la prédominance des particularismes nationaux, et cela davantage, lorsqu'il s'agit des politiques d'immigration, d'intégration, d'accès à la citoyenneté. Les propos de Charles Pasqua, alors Ministre de l'Intérieur, lors des débats sur les nouvelles mesures concernant la loi sur la nationalité, les contrôles d'identités et l'immigration sont à cet égard révélateurs: " Pour garder la maîtrise de son identité, la France entend définir par elle-même sa situation, la qualité, l'origine de ceux qui sont ou seront associés à la 
communauté nationale dans l'esprit des valeurs de sa République, dans le cadre de sa Constitution et dans le respect du droit international auquel elle a librement consenti "20.

En Allemagne, l'article 16 de la Loi Fondamentale concernant les demandeurs d'asile a constitué de même un sujet brûlant au début des années 1990. Les débats sur la suppression de cet article ont révélé des propos assez proches de ceux de l'ancien Ministre de l'Intérieur français: " il s'agit du droit d'autodétermination des peuples, droit qui prévaut sur le droit de séjour. Ce droit à l'autodétermination découle de la souveraineté de l'Etat ; c'est l'indépendance vis-à-vis de toute autorité étrangère, le pouvoir de décider de l'évolution politique, économique, sociale et culturelle de son propre pays "21.

En définitive, chacun des deux pays développe une argumentation dictée par sa conception propre d'un " mal " issu de la présence des étrangers : la France craint la non-identification des Français d'origine étrangère avec la communauté nationale; l'Allemagne soupçonne l'utilisation abusive du droit de séjour des demandeurs d'asile. S'y ajoute, en outre, un sentiment xénophobe qui prétend se justifier par la crise économique qui touche tous les pays européens. Au total, ce " mal " venu d'ailleurs conduit à affirmer un " droit à l'auto-détermination " qui paraphrase en quelque sorte, au sein de l'Europe, celui des minorités ou des peuples opprimés. Cette évolution accroît les tensions déjà existantes entre les populations immigrées, les populations majoritaires et les Etats.

39 L'Union européenne représente une idée d'ouverture, une autre conception de l'universalité que celle des Etats-Nations, perçue comme particulière. L'idée d'universalité correspondant à l'Europe serait, selon les militants de l'immigration, celle d'un espace où les étrangers, résidents en Europe, ou même les citoyens perçus comme étrangers (du fait de leur nationalité d'origine perçue comme une identité ethnique, du fait de la couleur ou de la religion), s'inscriraient dans une pluralité des cultures, au même titre que celles se référant aux identités nationales traditionnelles. Imaginer une " communauté transnationale " issue de l'immigration apporterait un renfort aux sentiments nationalistes exprimés par les Etats membres face à l'immigration d'une part et à la construction européenne de l'autre. Mais en même temps, l'irrationalité des sentiments nationaux réduits à une appartenance ethnique s'oppose à la rationalité des institutions européennes qui, dans un souci d'harmonisation, définissent des normes juridiques dans ces domaines, notamment les Droits de l'Homme et le droit des minorités, domaines qui concernent les "étrangers de l'intérieur ".

40 La structuration des réseaux transnationaux traduit une européanisation de l'action politique des populations issues de l'immigration, encouragée par les institutions supranationales. Mais elle n'entraîne pas l'européanisation des revendications. Ces dernières restent liées aux Etats qui restent les seuls cadres de référence concrets de mobilisations et de négociations. Bien entendu, cette forme d'organisation qui transcende les frontières nationales met en évidence le principe des identifications multiples qui découlent de la logique de l'idée d'Europe politique. C'est précisément ce qui suscite les débats passionnés qui accompagnent sa construction, car il apporte un bouleversement au concept de citoyenneté lié à la nationalité et à l'identité politique, ainsi qu'à la territorialité de la participation, et marque par conséquence l'affaiblissement des Etats-Nations face à des revendications identitaires qui 
s'expriment à l'intérieur des frontières nationales. Mais, la non pertinence de l'EtatNation dans une Europe politique n'implique pas nécessairement l'affaiblissement de l'Etat, qui, lui, reste la force structurante de la construction européenne.

\section{NOTES}

1. Citations extraites de la conférence d'Ernest Renan à la Sorbonne en 1882 in E. Renan, Qu'estce qu'une nation?

2. J'emprunte l'expression à Pierre Muller in " Les mutations des politiques publiques européennes ", Pouvoirs, 69-1994, p. 64.

3. Cf. Esprit, Mars 1992.

4. La France accueille la majorité des Maghrébins (820 000 Algériens ; 516000 Marocains ; 200000 Tunisiens), et l'Allemagne le plus grand nombre de Turcs (1 700 000). Les Turcs (160 000) et les Marocains (123 000) forment le gros des communautés extra-européennes immigrées aux PaysBas, tandis que la Grande-Bretagne se caractérise par l'importance de ses populations d'origine indienne (689 000), antillaise (547 000) et pakistanaise (406 000) ; Sources SOPEMI-OCDE, Eurostat, INED (1990).

5. Cf. W. R. Brubaker, Citizenship and Nationhood in France and Germany, Cambridge MASS., Harvard University Press, 1992 ; L. Dumont, L'idéologie allemande. France-Allemagne et retour, Paris, Gallimard, 1991 ; D. Schnapper, La France de l'intégration. Sociologie de la nation en 1990, Paris, Gallimard, 1991.

6. C. Neveu, " Citoyenneté ou racisme en Europe : exception et complémentarité britanniques ", Revue Européenne des Migrations Internationales, vol. 10, n 1, 1994, pp. 95-109.

7. En 1990, 29\% des individus interrogés souhaitaient que les droits des immigrés fussent restreints. En 1992, ce taux est monté à 34\%. De même en $199160 \%$ des personnes auraient accepté l'arrivée des migrants de la Méditerranée avec des restrictions, et en 1993, seulement 46\% de l'opinion était d'accord. Eurobaromètre, décembre 1992.

8. Cf. Christian Lequesne, " La Commission européenne entre autonomie et dépendance ", Revue Française de Science Politique, vol. 46, n 3, juin 1996, pp. 389-408.

9. Pour la citoyenneté comme sentiment d'appartenance et citoyenneté comme engagement, voir Jean Leca " Individualisme et citoyenneté " in Pierre Birnbaum, Jean Leca (dir.), Sur l'individualisme, Paris, Presses de la FNSP, 1986, pp. 159-213.

10. Jean-Marc Ferry, " Pertinence du postnational ", Esprit, novembre 1991, pp. 80-94.

11. Jacques Lenoble, L'Europe au soir des siècles, Paris, Seuil, coll. Esprit, 1992, pp.17-39.

12. Yasemin Soysal, Limits of Citizenship. Migrants and Postnational Membership in Europe, University of Chicago Press, 1994, p. 143.

13. Alain Touraine, Qu'est-ce que la démocratie?, Paris, Fayard, 1994, pp. 102-103.

14. J. Habermas, op.cit., 1992.

15. En référence à l'ouvrage de P. B. Evans, D. Rueschemeyer \& T. Skocpol (eds), Bringing the State Back In, Cambridge University Press, 1985.

16. B. de Witte, " The European Community and its Minorities " in Bremann et al. (eds), Peoples and Minorities in International Law, Kluwer Academic Publishers, Pays-Bas, 1993, pp. 167-185.

17. Voir F. Sudre, Droit international et européen des droits de l'homme, Paris PUF, 1997, pp. 153-158. 
18. Art.29, § 1 de la Déclaration cité par F. Sudre, op.cit., p.154.

19. Art. 2 de la proposition de convention de 1991, cité par F. Sudre, op.cit., p.156.

20. Le Monde, 17 juin 1993, p.7.

21. Extrait du Bulletin d'Information sur le droit d'asile, du Centre d'Information et de Documentation de l'Ambassade de la République Fédérale d'Allemagne, juin 1993.

\section{RÉSUMÉS}

Dans cet article, Riva Kastoryano analyse les formes d'organisation des populations immigrées et l'expression de leurs identités collectives, et interroge dès lors les traditions politiques nationales à l'aune de la construction d'une nouvelle forme de l'agir politique, l'Europe. En observant la constitution de ces réseaux de solidarité transnationaux, l'auteur propose une analyse de l'européanisation de l'action politique des populations issues de l'immigration et montre comment cette européanisation encouragée par les institutions supranationales produit des effets au niveau de chaque Etat-nation. En problématisant la question de la citoyenneté autour d'une sociologie de ces réseaux qui transcendent les frontières nationales, l'auteur interroge avec pertinence la question des allégeances citoyennes.

Riva Kastoryano analyses how immigrant populations organise and how they express their collective identity. The author thereby questions national political traditions with regards to the construction of a new form of political action: Europe. The author offers an analysis of the Europeanization of political action for these immigrant populations by observing the constitution of transnational networks. This article shows how this Europeanization being encouraged by supranational institutions produces effects within each Nation-State. The author pertinently questions the idea of citizen allegiances by giving a problematic to the notion of citizenship through the sociology of these networks transcending national frontiers.

\section{INDEX}

Mots-clés : immigration, mobilité, réseaux transnationaux

Index géographique : Bruxelles, Europe, Union européenne

\section{AUTEUR}

\section{RIVA KASTORYANO}

Chargée de recherche au CERI/CNRS. 